TTP/99-54

DESY $/ 00-008$

hep-ph/9912503

December 1999

\title{
Summing up Subleading Sudakov Logarithms
}

\author{
J.H. Kühn \\ Institut für Theoretische Teilchenphysik, Universität Karlsruhe \\ 76128 Karlsruhe, Germany \\ A.A. Penin* \\ II. Institut für Theoretische Physik, Universität Hamburg \\ 22761 Hamburg, Germany \\ V.A. Smirnov \\ Nuclear Physics Institute of Moscow State University \\ 119899 Moscow, Russia
}

\begin{abstract}
We apply the strategy of regions within dimensional regularization to find functions involved in evolution equations which govern the asymptotic dynamics of the Abelian form factor and four-fermion amplitude in the $S U(N)$ gauge theory in the Sudakov limit up to the next-to-leading logarithmic approximation. The results are used for the analysis of the dominant electroweak corrections to the fermion-antifermion pair production in the $e^{+} e^{-}$annihilation at high energy.
\end{abstract}

PACS numbers: 12.38.Bx, 12.38.Cy, 12.15.Lk

*On leave from Institute for Nuclear Research of Russian Academy of Sciences, 117312 Moscow, Russia 


\section{Introduction}

The asymptotic behavior of various amplitudes in the Sudakov limit has been investigated in QED and QCD, with summation of the leading double [1, 2, 3, 4] and subleading [5, 6, 7, 8, 9, 10, 11 logarithms. Evolution equations that govern the dynamics of the amplitudes in the Sudakov limit have been obtained in refs. [6, 0, 8, 10]. In the present paper, we apply this approach to the next-to-leading analysis of the Abelian form factor and the four fermion amplitude in the $S U(N)$ gauge theory. We evaluate functions that enter the evolution equations in the next-to-leading logarithmic approximation by using, as an input, asymptotic expansions of one-loop diagrams. Solving these equations we sum up the leading and subleading Sudakov logarithms. The expansions of one-loop diagrams are obtained by use of the so-called generalized strategy of regions [12] (see also [13]) which enables us to systematically identify the nature of various contributions and the origin of logarithms. This strategy is based on expanding integrands of Feynman integrals in typical regions and extending the integration domains to the whole space of the loop momenta so that a crucial difference with respect to the standard approach [6, 7, 8, 9, 10] is the absence of cut-offs that specify the regions in individual terms of the expansions. This approach is applied within dimensional regularization [14] when all integrals without scale (not only massless vacuum integrals) are put to zero.

We apply our results for the subleading Sudakov logarithms to the analysis of the dominant electroweak corrections to the process of the fermion-antifermion pair production in the $e^{+} e^{-}$annihilation. In the standard model of weak interactions the $W$ and $Z$ bosons get their masses via the Higgs mechanism and the Sudakov logarithms naturally appear in the virtual electroweak corrections [15]. They grow rapidly with energy and become dominant in the $\mathrm{TeV}$ region available at the $\mathrm{LHC}$ or the Next Linear Collider. The analysis of the Sudakov corrections is thus of high importance for the next generation of accelerators. The leading and subleading electroweak Sudakov logarithms were discussed in [16, 17] in one loop approximation. The effect of higher order leading logarithms was estimated in [18] by computing, in a physical gauge, a contribution related to the multiple virtual $Z$ and $W$ bosons exchanges. A complete analysis of the leading logarithms in exclusive and inclusive electroweak processes was done in [19] on the basis of the infrared evolution equation approach. The subleading Sudakov logarithms, however, turn out to be significant and should be taken into account to get a reliable estimate of high order corrections.

The paper is organized as follows. In the next section, the Abelian form factor is analyzed in two typical Sudakov type regimes. The analysis is then extended in Section 3 to the fourfermion amplitude. The electroweak Sudakov corrections are considered in Section 4. We present our conclusions in the last section. 


\section{The Abelian form factor in the Sudakov limit}

The (vector) form factor which determines the amplitude of the fermion scattering in the external Abelian field in the Born approximation can be written as follows

$$
F_{B}=\bar{\psi}\left(p_{2}\right) \gamma_{\mu} \psi\left(p_{1}\right)
$$

where the four-vector index on the left hand side is suppressed, $p_{1}$ is incoming and $p_{2}$ is outgoing momentum.

There are two "standard" regimes of the Sudakov limit $s=\left(p_{1}-p_{2}\right)^{2} \rightarrow-\infty$ [1, 2]:

( $i$ ) On-shell massless fermions, $p_{1}^{2}=p_{2}^{2}=0$, and gauge bosons with a small non-zero mass $M^{2} \ll-s$. Let us choose, for convenience, $p_{1,2}=(Q / 2,0,0, \mp Q / 2)$ so that $2 p_{1} p_{2}=Q^{2}=-s$.

(ii) The massless gauge bosons and off-shell massless fermions $p_{1}^{2}=p_{2}^{2}=-M^{2}, M^{2} \ll-s$. We choose $p_{1}=\tilde{p}_{1,2}-\left(M^{2} / Q^{2}\right) \tilde{p}_{2,1}$, where $\tilde{p}_{1,2}$ are defined as $p_{1,2}$ in the regime $(i)$.

The asymptotics of the form factor in the Sudakov limit can be found by solving the corresponding evolution equation [6]. For the non-Abelian gauge theory, this equation was first derived in [7] by factorizing collinear logarithms in the axial gauge. In the first regime, it reads

$$
\frac{\partial}{\partial \ln Q^{2}} F=\left[\int_{M^{2}}^{Q^{2}} \frac{\mathrm{d} x}{x} \gamma(\alpha(x))+\zeta\left(\alpha\left(Q^{2}\right)\right)+\xi\left(\alpha\left(M^{2}\right)\right)\right] F
$$

Its solution is

$$
F=F_{0}\left(\alpha\left(M^{2}\right)\right) \exp \left\{\int_{M^{2}}^{Q^{2}} \frac{\mathrm{d} x}{x}\left[\int_{M^{2}}^{x} \frac{\mathrm{d} x^{\prime}}{x^{\prime}} \gamma\left(\alpha\left(x^{\prime}\right)\right)+\zeta(\alpha(x))+\xi\left(\alpha\left(M^{2}\right)\right)\right]\right\} .
$$

A generalization of eq. (2) to the regime (ii) was found in [10]:

$$
\frac{\partial}{\partial \ln Q^{2}} F=\left[\int_{M^{4} / Q^{2}}^{Q^{2}} \frac{\mathrm{d} x}{x} \gamma(\alpha(x))+\zeta\left(\alpha\left(Q^{2}\right)\right)+\zeta^{\prime}\left(\alpha\left(M^{4} / Q^{2}\right)\right)+\xi\left(\alpha\left(M^{2}\right)\right)\right] F
$$

Its solution is

$$
\begin{aligned}
F= & F_{0}\left(\alpha\left(M^{2}\right)\right) \exp \left\{\int_{M^{2}}^{Q^{2}} \frac{\mathrm{d} x}{x}\left[\int_{M^{2}}^{x} \frac{\mathrm{d} x^{\prime}}{x^{\prime}} \gamma\left(\alpha\left(x^{\prime}\right)\right)+\zeta(\alpha(x))+\xi\left(\alpha\left(M^{2}\right)\right)\right]\right. \\
& \left.+\int_{M^{4} / Q^{2}}^{M^{2}} \frac{\mathrm{d} x}{x}\left[\int_{x}^{M^{2}} \frac{\mathrm{d} x^{\prime}}{x^{\prime}} \gamma\left(\alpha\left(x^{\prime}\right)\right)+\zeta^{\prime}(\alpha(x))\right]\right\}
\end{aligned}
$$

The functions $F_{0}$ and $\xi$ are, generally, different in the two regimes. We are interested in the next-to-leading logarithms. Therefore we should keep renormalization group corrections to the leading logarithmic approximation as well as single infrared and renormalization group logarithms. In this approximation, the form factor $(i)$ takes the form

$$
F=F_{0}(\alpha) \exp \left[\int_{M^{2}}^{Q^{2}} \frac{\mathrm{d} x}{x} \int_{M^{2}}^{x} \frac{\mathrm{d} x^{\prime}}{x^{\prime}} \gamma\left(\alpha\left(x^{\prime}\right)\right)+(\zeta(\alpha)+\xi(\alpha)) \ln \left(Q^{2} / M^{2}\right)\right]
$$


and, in the regime $(i i)$, we have

$$
\begin{aligned}
F=F_{0}(\alpha) \exp \left\{\int_{M^{2}}^{Q^{2}} \frac{\mathrm{d} x}{x} \int_{M^{2}}^{x} \frac{\mathrm{d} x^{\prime}}{x^{\prime}} \gamma\left(\alpha\left(x^{\prime}\right)\right)+\int_{M^{4} / Q^{2}}^{M^{2}} \frac{\mathrm{d} x}{x} \int_{x}^{M^{2}} \frac{\mathrm{d} x^{\prime}}{x^{\prime}} \gamma\left(\alpha\left(x^{\prime}\right)\right)\right. \\
\left.+\left(\zeta(\alpha)+\zeta^{\prime}(\alpha)+\xi(\alpha)\right) \ln \left(Q^{2} / M^{2}\right)\right\} .
\end{aligned}
$$

All the functions in the exponent have to be computed in one loop, and the one loop running of $\alpha$ in the argument of the $\gamma$ function should be taken into account. Note that in the nextto-leading order we cannot separate the functions $\zeta$ and $\xi$ but we will see that $\zeta^{\prime}$ vanishes in one loop.

In the covariant gauge, the self energy insertions to the external fermion lines do not give $Q$-dependent contributions. The one loop calculation of the vertex correction gives

$$
F=\frac{\alpha}{2 \pi} C_{F}\left(-V_{0}+2 V_{1}+2(1-2 \epsilon) V_{2}-V_{2}^{\prime}\right) F_{B}
$$

where $C_{F}=\left(N^{2}-1\right) /(2 N)$ is the quadratic Casimir operator of the fundamental representation of $S U(N)$ group and the functions involved in are given by

$$
\begin{aligned}
& \int \frac{\mathrm{d}^{d} k}{\left(k^{2}-2 p_{1} k\right)\left(k^{2}-2 p_{2} k\right)\left(k^{2}-M^{2}\right)}=i \pi^{d / 2} e^{-\gamma_{\mathrm{E}} \epsilon} s^{-1} V_{0} \\
& \int \frac{\mathrm{d}^{d} k k_{\mu}}{\left(k^{2}-2 p_{1} k\right)\left(k^{2}-2 p_{2} k\right)\left(k^{2}-M^{2}\right)}=i \pi^{d / 2} e^{-\gamma_{\mathrm{E}} \epsilon} s^{-1}\left(p_{1}+p_{2}\right)_{\mu} V_{1} \\
& \int \frac{\mathrm{d}^{d} k k_{\mu} k_{\nu}}{\left(k^{2}-2 p_{1} k\right)\left(k^{2}-2 p_{2} k\right)\left(k^{2}-M^{2}\right)}=i \pi^{d / 2} e^{-\gamma_{\mathrm{E}} \epsilon}\left[g_{\mu \nu} V_{2}+\frac{p_{1_{\mu}} p_{2_{\nu}}+(\mu \leftrightarrow \nu)}{s} V_{2}^{\prime}\right]
\end{aligned}
$$

in the regime $(i)$. The corresponding relations for the regime (ii) are obtained from eq. (9) by the following substitution

$$
\left(k^{2}-2 p_{1} k\right)\left(k^{2}-2 p_{2} k\right)\left(k^{2}-M^{2}\right) \rightarrow\left(k^{2}-2 p_{1} k-M^{2}\right)\left(k^{2}-2 p_{2} k-M^{2}\right) k^{2},
$$

with another set of the functions $V$ involved. We omit $i 0$ in $k^{2}-2 p_{1} k+i 0$, etc. for brevity. Here $\gamma_{\mathrm{E}}$ is the Euler constant. We work in dimensional regularization [14] with $d=4-2 \epsilon$. We also usually omit the factor $\left(\mu^{2}\right)^{\epsilon}$ per loop and write it down only in the argument of the renormalization group logarithm. Note that only the tensor structures giving unsuppressed contributions to the form factor are kept in the representation of the third integral.

To expand these integrals in the limit $Q^{2} \gg M^{2}$ we apply a generalized strategy of regions formulated in [12 and discussed using characteristic two-loop examples in [13]:

- Consider various regions of the loop momenta and expand, in every region, the integrand in Taylor series with respect to the parameters that are there considered small;

- Integrate the expanded integrand over the whole integration domain of the loop momenta; 
- Put to zero any scaleless integral.

The following regions happen to be typical in the Sudakov limit [20]:

$$
\begin{aligned}
\text { hard (h): } & k \sim Q, \\
\text { 1-collinear (1c): } & k_{+} \sim Q, k_{-} \sim M^{2} / Q, \underline{k} \sim M, \\
\text { 2-collinear (2c): } & k_{-} \sim Q, k_{+} \sim M^{2} / Q, \underline{k} \sim M, \\
\text { soft (s): } & k \sim M, \\
\text { ultrasoft (us): } & k \sim M^{2} / Q .
\end{aligned}
$$

Here $k_{ \pm}=k_{0} \pm k_{3}, \underline{k}=\left(k_{1}, k_{2}\right)$. We mean by $k \sim Q$, etc. that any component of $k_{\mu}$ is of order $Q$.

Keeping the leading power in the expansion in the limit $Q^{2} / M^{2} \rightarrow \infty$ we have in the regime $(i)$ 凹

$$
\begin{aligned}
V_{0}^{h}=\frac{1}{\epsilon^{2}}-\frac{1}{\epsilon} \ln (-s) & +\frac{1}{2} \ln ^{2}(-s)-\frac{\pi^{2}}{12} \\
V_{0}^{c}=-\frac{1}{\epsilon^{2}}+\frac{1}{\epsilon} \ln (-s) & -\ln \left(M^{2}\right) \ln (-s)+\frac{1}{2} \ln ^{2}\left(M^{2}\right)+\frac{5 \pi^{2}}{12} \\
V_{0}=\frac{1}{2} \ln ^{2}\left(\frac{-s}{M^{2}}\right)+\frac{\pi^{2}}{3} & \\
V_{1}^{h} & =-\frac{1}{\epsilon}+\ln (-s)-2 \\
V_{1}^{c} & =\frac{1}{\epsilon}-\ln \left(M^{2}\right)+1 \\
V_{1} & =\ln \left(\frac{-s}{M^{2}}\right)-1 ; \\
V_{2}=V_{2}^{h} & =\frac{1}{4}\left(\frac{1}{\epsilon}-\ln \left(\frac{-s}{\mu^{2}}\right)+3\right) \\
V_{2}^{\prime}=V_{2}^{\prime h} & =\frac{1}{2} .
\end{aligned}
$$

We denote by the index $c$ the sum of the $1 \mathrm{c}$ and $2 \mathrm{c}$ contributions. The pole in eq. (13) is of ultraviolet nature. It is not canceled by the collinear or ultrasoft contributions. Note that the corresponding ultraviolet renormalization group logarithm $\ln \left(-s / \mu^{2}\right)$ contributes only to the function $\zeta$.

\footnotetext{
${ }^{1}$ In fact we do not need, in the LO and NLO, finite parts present in these and similar results. They would be, however, needed for the NNLO calculations.
} 
The hard part in the regime (ii) is the same. The new ingredients read

$$
\begin{aligned}
& V_{0}^{c}=-\frac{2}{\epsilon^{2}}+\frac{2}{\epsilon} \ln \left(M^{2}\right)-\ln ^{2}\left(M^{2}\right)+\frac{\pi^{2}}{6} \\
& V_{0}^{u s}= \frac{1}{\epsilon^{2}}+\frac{1}{\epsilon}\left(\ln (-s)-2 \ln \left(M^{2}\right)\right)+\frac{1}{2} \ln ^{2}(-s) \\
&-2 \ln \left(M^{2}\right) \ln (-s)+2 \ln \left(M^{2}\right)+\frac{\pi^{2}}{4} \\
& V_{0}= \ln ^{2}\left(\frac{-s}{M^{2}}\right)+\frac{\pi^{2}}{3} \\
& V_{1}^{c}= \frac{1}{\epsilon}+2-\ln \left(M^{2}\right) \\
& V_{1}=\ln \left(\frac{-s}{M^{2}}\right) .
\end{aligned}
$$

Soft regions generate only zero contributions (at least in the leading power).

From the one-loop result we find

$$
\gamma(\alpha)=-C_{F} \frac{\alpha}{2 \pi} .
$$

Moreover it is clear from the above expressions that in the regime $(i)$ the total double logarithms of $Q$ come from the hard region while in the regime (ii) one half of the double logarithmic contribution comes from the ultrasoft region. This explicitly determines the scale of the coupling constant in the second order logarithmic derivative of the form factor in $Q$. It is $Q$ in the regime $(i)$ and $M$ in the regime (ii). Furthermore, all the $Q$ dependent terms in the ultrasoft contribution of eq. (14) are related to the $\gamma$ term and therefore $\zeta^{\prime}(\alpha)=0$. At the same time we cannot distinguish, in the one loop approximation, the contribution to the functions $\zeta$ and $\xi$ coming from the collinear region because this region includes both $Q$ and $M$ scales. For the sum of these functions we find

$$
\zeta(\alpha)+\xi(\alpha)=3 C_{F} \frac{\alpha}{4 \pi} .
$$

To complete the $Q$-independent part of the one loop corrections to the form factor one has to include the fermion wave function renormalization determined by the self energy insertions to the external lines. In the regime $(i)$ this brings the factor

$$
1+C_{F} \frac{\alpha}{4 \pi}\left(-\frac{1}{\epsilon}+\ln \left(\frac{M^{2}}{\mu^{2}}\right)+\frac{1}{2}\right)
$$

and in the regime (ii) this gives

$$
1+C_{F} \frac{\alpha}{4 \pi}\left(-\frac{1}{\epsilon}+\ln \left(\frac{M^{2}}{\mu^{2}}\right)-1\right) .
$$


The ultraviolet poles of eqs. (18, 19) cancel the ultraviolet pole in the first line of eq. (13) due to Ward identity and nonrenormalization property of the conserved vector current. Finally, in the NLO logarithmic approximation, we find the form factor $(i)$ to be

$$
\begin{aligned}
F= & F_{B}\left(1-C_{F} \frac{\alpha}{2 \pi}\left(\frac{7}{2}+\frac{2 \pi^{2}}{3}\right)\right) \exp \left\{\frac { C _ { F } } { 2 \pi } \left[-\int_{M^{2}}^{Q^{2}} \frac{\mathrm{d} x}{x} \int_{M^{2}}^{x} \frac{\mathrm{d} x^{\prime}}{x^{\prime}} \alpha\left(x^{\prime}\right)\right.\right. \\
& \left.\left.+3 \alpha \ln \left(Q^{2} / M^{2}\right)\right]\right\}
\end{aligned}
$$

and the form factor $(i i)$ to be

$$
\begin{aligned}
F= & F_{B}\left(1-C_{F} \frac{\alpha}{2 \pi}\left(1+\frac{2 \pi^{2}}{3}\right)\right) \exp \left\{\frac { C _ { F } } { 2 \pi } \left[-\int_{M^{2}}^{Q^{2}} \frac{\mathrm{d} x}{x} \int_{M^{2}}^{x} \frac{\mathrm{d} x^{\prime}}{x^{\prime}} \alpha\left(x^{\prime}\right)\right.\right. \\
& \left.\left.-\int_{M^{4} / Q^{2}}^{M^{2}} \frac{\mathrm{d} x}{x} \int_{x}^{M^{2}} \frac{\mathrm{d} x^{\prime}}{x^{\prime}} \alpha\left(x^{\prime}\right)+3 \alpha \ln \left(Q^{2} / M^{2}\right)\right]\right\} .
\end{aligned}
$$

The single logarithmic term in the exponents of eqs. (20, 21) has the following decomposition

$$
3 \ln \left(Q^{2} / M^{2}\right)=4 \ln \left(Q^{2} / M^{2}\right)_{(I R)}-\ln \left(Q^{2} / M^{2}\right)_{(R G)},
$$

where we explicitly separate the infrared and renormalization group logarithms which are related to $V_{1}$ and $V_{2}$ integrals correspondingly. These logarithms are of essentially different nature (see a discussion below). Eqs. (20, 21) are in agreement with the result of refs. [9, 10].

It is useful to distinguish the soft and collinear poles in $\epsilon$ (resulting in logarithms) in the hard contribution. The collinear logarithms in a physical (Coulomb or axial) gauge originate only from the self energy insertions into the external fermion lines [4, 6, 0, 8, 21, 22, 23] and therefore are universal i.e. independent of specific processes. In contrast to the soft divergences (i.e. infrared divergences that are local in momentum space), the divergences of this type arise from the integration over angle variables. Consider, for example, the integral $V_{1}$. The power counting tells us that there is no soft divergence in this integral (we have $k^{5}$ in the numerator and only $k^{4}$ in the denominator). However, for non-zero $k$ with $k^{2} \sim 0$ which is collinear to $p_{1}$ or $p_{2}$ (when $p_{1} k \sim 0$ or $p_{2} k \sim 0$ ), the integrand blows up. This follows from the fact that the factor $1 /\left(\left(k^{2}-2 p k\right) k^{2}\right)$, with any $p^{2}=0$, generates collinear divergences. When integrating this factor in $k_{0}$, take residues in the upper half plane. For example, taking a residue at $k_{0}=-|\vec{k}|$ leads to an integral with $1 /(p k)=1 /\left(p^{0}|\vec{k}|(1-\cos \theta)\right)$ where $\theta$ is the angle between the spatial components. Thus, for small $\theta$, we have a divergent integration over angles $d \cos \theta /(1-\cos \theta) \sim d \theta / \theta$. The second residue generates a similar divergent behaviour - this can be seen by the change $k \rightarrow p-k$.

Within the method of regions the total divergence of the collinear region in general cancels both the soft and collinear poles of the hard part. Hence it is not straightforward

\footnotetext{
${ }^{2}$ Although this logarithm originates from the integration over the virtual momentum region between $M$ and $Q$ scales and does not depend on $\mu$ we call it "renormalization group" one because it is directly related and can be read off the renormalization group properties of the Abelian vertex and the fermion wave function.
} 
to separate the collinear logarithms. Let us note that the collinear divergences (but not the soft ones) can be regularized by introducing a finite fermion mass. The reason is that the light-like vector $k$ cannot be collinear to the space-like vector $p_{i}$ in this case. This enables us to distinguish the soft and collinear poles. Introducing a small fermion mass $m$ but keeping zero gauge boson mass we get the collinear poles in the hard part canceled by the poles of the contribution from the collinear region leaving the logarithms while the soft poles in the hard part are not canceled. Thus one can determine the origin of the poles in the hard part and therefore the origin of the logarithms. For example, in the hard part of the integral $V_{1}$ the pole is of the collinear origin since it is canceled in the regime $m \neq 0, M=0$ by

$$
V_{1}^{c}=\frac{1}{\epsilon}-\ln \left(m^{2}\right)+2 .
$$

Thus the single infrared logarithm in eq. (22) is of collinear origin and therefore is universal. We should emphasize that this is not true for the renormalization group logarithm of this equation which depends on a specific amplitude and a model. For example it is different for the scalar form factor or for the vector form factor in a model with an additional Yukawa interaction of the fermions with the scalar bosons.

A less trivial example is the integral $V_{0}$. In the hard part of $V_{0}$ in this regime, the collinear part of the double pole is canceled by

$$
V_{0}^{c}=-\frac{1}{\epsilon^{2}}+\frac{1}{\epsilon} \ln \left(m^{2}\right)-\frac{1}{2} \ln ^{2}\left(m^{2}\right)-\frac{\pi^{2}}{12}
$$

and transforms into the logarithm of $s / m^{2}$ but the soft single pole is left

$$
V_{0}=-\frac{1}{\epsilon} \ln \left(\frac{-s}{m^{2}}\right)+\ln \left(\frac{-s}{m^{2}}\right) \ln (-s)-\frac{1}{2} \ln ^{2}\left(\frac{-s}{m^{2}}\right)-\frac{\pi^{2}}{6} .
$$

\section{The four fermion amplitude}

We study the limit of the fixed-angle scattering when all the invariant energy and momentum transfers of the process are much larger than the typical mass scale of internal particles $|s| \sim|t| \sim|u| \gg M^{2}$. Besides the extra kinematical variable the analysis of the four fermion amplitude is more complicated by the presence of different "color" and Lorentz structures. The Born amplitude, for example, can be expanded in the basis of color/chiral amplitudes

$$
A_{B}=\frac{i g^{2}}{s} A^{\lambda}=\frac{i g^{2}}{s} T_{F}\left(-\frac{1}{N}\left(A_{L L}^{d}+A_{L R}^{d}\right)+A_{L L}^{c}+A_{L R}^{c}+(L \leftrightarrow R)\right),
$$

where

$$
\begin{aligned}
A^{\lambda} & =\bar{\psi}_{2}\left(p_{2}\right) t^{a} \gamma_{\mu} \psi_{1}\left(p_{1}\right) \bar{\psi}_{4}\left(p_{4}\right) t^{a} \gamma_{\mu} \psi_{3}\left(p_{3}\right) \\
A_{L L}^{d} & =\bar{\psi}_{2}^{i} \gamma_{\mu} \psi_{1 L}^{i} \bar{\psi}_{4 L}^{j} \gamma_{\mu} \psi_{3 L}^{j} \\
A_{L R}^{c} & =\bar{\psi}_{2}^{j} \gamma_{\mu} \gamma_{1 L}^{i} \bar{\psi}_{4 R}^{i} \gamma_{\mu} \psi_{3 R}^{j}
\end{aligned}
$$


and so on. Here $t^{a}$ is the $S U(N)$ generator, $p_{1}, p_{3}$ are incoming and $p_{2}, p_{4}$ outgoing momenta so that $t=\left(p_{1}-p_{4}\right)^{2}$ and $u=\left(p_{1}+p_{3}\right)^{2}=-(s+t)$. For the moment we consider a parity conserving theory. Hence only two chiral amplitudes are independent, for example, $L L$ and $L R$ [5. Similarly only two color amplitudes are independent, for example, $\lambda$ and $d$.

Let us first compute the one loop corrections in the regime $(i)$. The total contribution of the vertex type diagrams is

$$
\frac{\alpha}{\pi}\left(C_{F}\left(-V_{0}+2 V_{1}\right)+\frac{C_{A}}{2} V_{0}+\ldots\right) A_{B}
$$

where the ellipsis stands for the contribution without infrared logarithms. In the vertex correction involving the gauge boson selfcoupling, a contribution of the form (23) appears with $m$ replaced by $M$ and we have used the fact that the pole (logarithmic) term of $V_{1}^{c}$ is the same both in eqs. (23) and (12).

The direct (uncrossed) box gives

$$
\begin{aligned}
& \frac{i g^{2}}{s} \frac{\alpha}{4 \pi}\left[\left(5 B_{0}+3 B_{1}+4 B_{2}-3 B_{3}-6 B_{4}+2 B_{5}\right)\right. \\
& \times\left\{\left(C_{F}-\frac{T_{F}}{N}\right) \bar{\psi}_{2} t^{a} \gamma_{\mu} \psi_{1} \bar{\psi}_{4} t^{a} \gamma_{\mu} \psi_{3}+C_{F} \frac{T_{F}}{N} \bar{\psi}_{2} \gamma_{\mu} \psi_{1} \bar{\psi}_{4} \gamma_{\mu} \psi_{3}\right\} \\
& -\left(3 B_{0}+B_{1}-B_{3}-2 B_{4}+2 B_{5}\right) \\
& \left.\times\left\{\left(C_{F}-\frac{T_{F}}{N}\right) \bar{\psi}_{2} t^{a} \gamma_{\mu} \gamma_{5} \psi_{1} \bar{\psi}_{4} t^{a} \gamma_{\mu} \gamma_{5} \psi_{3}+C_{F} \frac{T_{F}}{N} \bar{\psi}_{2} \gamma_{\mu} \gamma_{5} \psi_{1} \bar{\psi}_{4} \gamma_{\mu} \gamma_{5} \psi_{3}\right\}\right],
\end{aligned}
$$

where $T_{F}=1 / 2$ is the index of the fundamental representation,

$$
\begin{array}{lll}
B_{0}=-i s J(1,1,1,1,1), & B_{1}=\text { st } J(1,2,1,1,1), & B_{2}=\text { st } J(1,1,2,1,1), \\
B_{3}=\text { st } J(1,3,1,1,2), & B_{4}=\text { st } J(2,1,2,1,2), & B_{5}=s^{2} J(1,1,2,2,2),
\end{array}
$$

and the functions $J\left(a_{1}, a_{2}, a_{3}, a_{4}, n\right)$ are proportional to scalar box integrals with shifted dimensions:

$$
\begin{aligned}
& J\left(a_{1}, a_{2}, a_{3}, a_{4}, n\right)=i^{-a_{1}-a_{2}-a_{3}-a_{4}-1+d / 2+n} \prod_{i}\left(a_{i}-1\right) ! \\
& \times \int \frac{\mathrm{d}^{d+n} k}{\left(k^{2}\right)^{a_{1}}\left(k^{2}-2 p_{1} k-m^{2}\right)^{a_{2}}\left(k^{2}-2 p_{2} k-m^{2}\right)^{a_{3}}\left(k^{2}-2\left(p_{1}-p_{4}\right) k+t\right)^{a_{4}}} .
\end{aligned}
$$

We apply the generalized strategy of regions to obtain a table of asymptotic expansions of the basic integrals in the leading power. Note that, besides the hard contribution, there are two groups of the 1c, $2 \mathrm{c}$ and us-contributions corresponding to two choices of the loop momentum when it is considered to be the momentum flowing through one of the two gauge

\footnotetext{
${ }^{3} \mathrm{~A}$ translation to the SM with the different structures of the amplitudes for different chiralities will be considered in Section 4.
} 
boson lines of the given box. Keeping terms with the leading and subleading logarithms we have

$$
\begin{gathered}
B_{0}=B_{5}=B_{1}-B_{3}-2 B_{4}=0, \\
B_{2}^{h}(s, t)=-\frac{1}{\epsilon^{2}}+\frac{1}{\epsilon} \ln (-t)+\frac{1}{2} \ln ^{2}(-s)-\ln (-s) \ln (-t), \\
B_{2}^{c}(s, t)=\frac{1}{\epsilon^{2}}-\frac{1}{\epsilon} \ln (-t)-\ln \left(M^{2}\right) \ln (-t)-\frac{1}{2} \ln ^{2}\left(M^{2}\right), \\
B_{2}(s, t)=\frac{1}{2} \ln ^{2}\left(\frac{-s}{M^{2}}\right)+\ln \left(\frac{-s}{M^{2}}\right) \ln \left(\frac{t}{s}\right),
\end{gathered}
$$

and the direct box contribution reads

$$
-\frac{i g^{2}}{s} \frac{\alpha}{\pi} B_{2}(s, t)\left(\left(C_{F}-\frac{T_{F}}{N}\right) A^{\lambda}+C_{F} \frac{T_{F}}{N} A^{d}\right) .
$$

The crossed box contribution can be obtained in the same way:

$$
\frac{i g^{2}}{s} \frac{\alpha}{\pi} B_{2}(s, u)\left(\left(C_{F}-\frac{T_{F}}{N}-\frac{C_{A}}{2}\right) A^{\lambda}+C_{F} \frac{T_{F}}{N} A^{d}\right)
$$

where $C_{A}=N$ is the quadratic Casimir operator of the adjoint representation. The rest of the one loop logarithmic contributions from the vertex corrections and the self-energy insertions are of the renormalization group nature. In addition to the vertex and external fermion selfenergy contributions considered in the previous section the renormalization group logarithms set the scale of $g$ in the Born amplitude to be $Q$.

The total one loop correction in the logarithmic approximation reads

$$
\begin{aligned}
\frac{i g^{2}\left(Q^{2}\right)}{s} \frac{\alpha}{2 \pi}\left[\left\{-C_{F} \ln ^{2}\left(\frac{-s}{M^{2}}\right)\right.\right. & \left.+\left(3 C_{F}-C_{A} \ln \left(\frac{u}{s}\right)+2\left(C_{F}-\frac{T_{F}}{N}\right) \ln \left(\frac{u}{t}\right)\right) \ln \left(\frac{-s}{M^{2}}\right)\right\} A^{\lambda} \\
+ & \left.\left\{2 \frac{C_{F} T_{F}}{N} \ln \left(\frac{u}{t}\right) \ln \left(\frac{-s}{M^{2}}\right)\right\} A^{d}\right] .
\end{aligned}
$$

Note that the next-to-leading logarithms do not depend on chirality and are the same both for the $L L$ and $L R$ amplitudes.

Now the collinear logarithms can be separated from the total one-loop correction. For each fermion-antifermion pair, they form the exponential factor found in the previous section (eq. (20)). This factor in addition incorporates the renormalization group logarithms which are not absorbed by changing the normalization scale of the gauge coupling. The rest of the single logarithms in eq. (36) is of the soft nature. Let us denote by $\tilde{A}$ the amplitude with the collinear logarithms factored out. It can be represented as a vector in the basis $A^{\lambda}, A^{d}$ and satisfies the following evolution equation [8, 24]

$$
\frac{\partial}{\partial \ln Q^{2}} \tilde{A}=\chi\left(\alpha\left(Q^{2}\right)\right) \tilde{A},
$$


where $\chi$ is the matrix of the "soft" anomalous dimensions. From eq. (37) we find the elements of this matrix to be, in units of $\alpha /(4 \pi)$,

$$
\begin{aligned}
& \chi_{\lambda \lambda}=-2 C_{A} \ln \left(\frac{u}{s}\right)+4\left(C_{F}-\frac{T_{F}}{N}\right) \ln \left(\frac{u}{t}\right), \\
& \chi_{\lambda d}=4 \frac{C_{F} T_{F}}{N} \ln \left(\frac{u}{t}\right) \\
& \chi_{d \lambda}=4 \ln \left(\frac{u}{t}\right) \\
& \chi_{d d}=0 .
\end{aligned}
$$

The solution of eq. (37) reads

$$
\tilde{A}=A_{1}^{0}\left(\alpha\left(M^{2}\right)\right) \exp \left[\int_{M^{2}}^{Q^{2}} \frac{\mathrm{d} x}{x} \chi_{1}(\alpha(x))\right]+A_{2}^{0}\left(\alpha\left(M^{2}\right)\right) \exp \left[\int_{M^{2}}^{Q^{2}} \frac{\mathrm{d} x}{x} \chi_{2}(\alpha(x))\right]
$$

where $\chi_{i}$ are eigenvalues of the $\chi$ matrix and $A_{i}^{0}$ are $Q$-independent vectors. Note that in higher orders the matrices $\chi$ for different values of $Q$ do not commute and the solution is given by the path-ordered exponent [8].

Equations (33) imply that only the hard parts contribute to eq. (37). This fixes the scale of $\alpha$ in this equation to be $Q$. By this reason the matrix (38) is the same in the regime $(i i)$. Hence in the next-to-leading logarithmic approximation the difference between the corrections to the four-quark amplitude in the regimes $(i)$ and $(i i)$ is the one between the factors (20) and (21).

In the Abelian case, there are no different color amplitudes and there is only one anomalous dimension

$$
\chi=4 \ln \left(\frac{u}{t}\right) .
$$

\section{Sudakov logarithms in electroweak processes}

We are interested in the process $f^{\prime} \bar{f}^{\prime} \rightarrow f \bar{f}$. In the Born approximation, its amplitude is of the following form

$$
A_{B}=\frac{i g^{2}}{s} \sum_{I, J=L, R}\left(T_{f^{\prime}}^{3} T_{f}^{3}+t_{W}^{2} \frac{Y_{f^{\prime}} Y_{f}}{4}\right) A_{I J}^{f^{\prime} f}
$$

where

$$
A_{I J}^{f^{\prime} f}=\bar{f}_{I}^{\prime} \gamma_{\mu} f_{I}^{\prime} \bar{f}_{J} \gamma_{\mu} f_{J}
$$

$t_{W}=\tan \theta_{W}$ with $\theta_{W}$ being the Weinberg angle and $T_{f}\left(Y_{f}\right)$ is the isospin (hypercharge) of the fermion which depends on the fermion chirality.

To analyze the electroweak correction to the above process we use the approximation with the $W$ and $Z$ bosons of the same mass $M$ and massless quarks and leptons. A fictitious photon mass $\lambda$ has to be introduced to regularize the infrared divergences. Let us consider first the equal mass case $\lambda=M$, where we can work in terms of the fields of unbroken phase 
and the result of Sects. 2 and 3 for the regime $(i)$ can be directly applied by projecting on a relevant initial/final state. For each fermion-antifermion pair the factor (20) takes the form

$$
\exp \left[-\left(T_{f}\left(T_{f}+1\right)+t_{W}^{2} \frac{Y_{f}^{2}}{4}\right)(L(s)-3 l(s))\right],
$$

where

$$
\begin{gathered}
L(s)=\frac{g^{2}}{16 \pi^{2}} \ln ^{2}\left(\frac{-s}{M^{2}}\right), \\
l(s)=\frac{g^{2}}{16 \pi^{2}} \ln \left(\frac{-s}{M^{2}}\right),
\end{gathered}
$$

and we neglect the running of the coupling constant in the integral in eq. (20) but fix the scale of the coupling $g$ and $t_{W} g$ in the double logarithmic contribution to be $Q$. The soft anomalous dimension for $I$ and/or $J=R$ is Abelian and, in units of $g^{2} /\left(16 \pi^{2}\right)$, reads

$$
\chi=t_{W}^{2} Y_{f^{\prime}} Y_{f} \ln \left(\frac{u}{t}\right)
$$

The matrix of the soft anomalous dimensions for $I=J=L$ is a sum of the Abelian and non-Abelian parts

$$
\begin{aligned}
\chi_{\lambda \lambda} & =-4 \ln \left(\frac{u}{s}\right)+\left(t_{W}^{2} Y_{f^{\prime}} Y_{f}+2\right) \ln \left(\frac{u}{t}\right), \\
\chi_{\lambda d} & =\frac{3}{4} \ln \left(\frac{u}{t}\right) \\
\chi_{d \lambda} & =4 \ln \left(\frac{u}{t}\right) \\
\chi_{d d} & =t_{W}^{2} Y_{f^{\prime}} Y_{f} \ln \left(\frac{u}{t}\right) .
\end{aligned}
$$

The photon is however massless, and the corresponding infrared divergent contributions should be accompanied by the real soft photon radiation integrated to some resolution energy $\omega_{\text {res }}$ to get an infrared safe cross section independent on an auxiliary photon mass. At the same time the massive gauge bosons are supposed to be detected as separate particles. In practice, the resolution energy is much less than the $W(Z)$ boson mass so the soft photon emission is of the QED nature. This cancels the infrared singularities of the QED virtual correction. We should therefore separate the QED virtual correction from the complete result computed with the photon of some mass $\lambda$ and then evaluate the QED virtual corrections together with the real soft photon radiation effects with vanishing $\lambda$. It is convenient to subtract the QED contribution computed with the photon of the mass $M$ from the obtained result for the virtual corrections and then take the limit $\lambda \rightarrow 0$ for the sum of QED virtual and real photon contributions to the total amplitude. In the language of the approach of ref. [19], this prescription means that we use the auxiliary photon mass $\lambda$ as a variable of the evolution equation below the scale $M$ and the subtraction fixes a relevant initial condition 
for this differential equation. This leads to a modification of the factor (43) and the soft anomalous dimensions (45, 47).

The common factor for each fermion-antifermion pair becomes

$$
\exp \left[-\left(T_{f}\left(T_{f}+1\right)+t_{W}^{2} \frac{Y_{f}^{2}}{4}-s_{W}^{2} Q_{f}^{2}\right)(L(s)-3 l(s))\right],
$$

where $s_{W}=\sin \theta_{W}$. Then we have

$$
\chi=\left(t_{W}^{2} Y_{f^{\prime}} Y_{f}-4 s_{W}^{2} Q_{f^{\prime}} Q_{f}\right) \ln \left(\frac{u}{t}\right),
$$

and the matrix of the soft anomalous dimension for $I=J=L$ is

$$
\begin{aligned}
\chi_{\lambda \lambda} & =-4 \ln \left(\frac{u}{s}\right)+\left(t_{W}^{2} Y_{f^{\prime}} Y_{f}-4 s_{W}^{2} Q_{f^{\prime}} Q_{f}+2\right) \ln \left(\frac{u}{t}\right) \\
\chi_{\lambda d} & =\frac{3}{4} \ln \left(\frac{u}{t}\right) \\
\chi_{d \lambda} & =4 \ln \left(\frac{u}{t}\right) \\
\chi_{d d} & =\left(t_{W}^{2} Y_{f^{\prime}} Y_{f}-4 s_{W}^{2} Q_{f^{\prime}} Q_{f}\right) \ln \left(\frac{u}{t}\right) .
\end{aligned}
$$

Now we can estimate the dominant one- and two-loop logarithmic corrections. The renormalization group logarithms which are not included into eq. (47) can be trivially taken into account by choosing the relevant scale of the coupling constants in the Born amplitude. At the same time, the remaining logarithmic corrections are of the main interest because they are supposed to dominate the (still unknown) total two-loop electroweak corrections. The one-loop leading and subleading logarithms can be directly obtained from eq. (36). The corresponding corrections to the chiral amplitudes read

$$
\begin{aligned}
& \left(\left[T_{f}\left(T_{f}+1\right)+t_{W}^{2} \frac{Y_{f}^{2}}{4}-s_{W}^{2} Q_{f}^{2}+\left(f \leftrightarrow f^{\prime}\right)\right]\left[T_{f^{\prime}}^{3} T_{f}^{3}+t_{W}^{2} \frac{Y_{f^{\prime}} Y_{f}}{4}\right](-L(s)+3 l(s))\right. \\
& +\left\{\left[-4 \ln \left(\frac{u}{s}\right)+2 \ln \left(\frac{u}{t}\right)+\ln \left(\frac{u}{t}\right) t_{W}^{2} Y_{f^{\prime}} Y_{f}\right] T_{f^{\prime}}^{3} T_{f}^{3}+\frac{3}{4} \ln \left(\frac{u}{t}\right) \delta_{I L} \delta_{J L}\right. \\
& \left.\left.+\ln \left(\frac{u}{t}\right)\left[t_{W}^{2} Y_{f^{\prime}} Y_{f}-4 s_{W}^{2} Q_{f^{\prime}} Q_{f}\right]\left[T_{f^{\prime}}^{3} T_{f}^{3}+t_{W}^{2} \frac{Y_{f^{\prime}} Y_{f}}{4}\right]\right\} l(s)\right) A_{I J}^{f^{\prime} f}
\end{aligned}
$$

where $\delta_{I L}=1$ for $I=L$ and zero otherwise. The two-loop leading (infrared) logarithms are determined by the second order term of the expansion of the double (soft $\times$ collinear) logarithmic part of the collinear factors (47). The corresponding corrections to the chiral amplitudes are

$$
\frac{1}{2}\left(T_{f}\left(T_{f}+1\right)+t_{W}^{2} \frac{Y_{f}^{2}}{4}-s_{W}^{2} Q_{f}^{2}+\left(f \leftrightarrow f^{\prime}\right)\right)^{2} L^{2}(s) A_{I J}^{f^{\prime} f}
$$


The two-loop next-to-leading logarithms are generated by the interference between the first order terms of the expansion of the double (soft $\times$ collinear) and single (soft+collinear+renormalization group) logarithmic exponents. The corresponding corrections to the chiral amplitudes are of the following form

$$
\begin{aligned}
& -\left[T_{f}\left(T_{f}+1\right)+t_{W}^{2} \frac{Y_{f}^{2}}{4}-s_{W}^{2} Q_{f}^{2}+\left(f \leftrightarrow f^{\prime}\right)\right] \\
& \times\left\{3\left[T_{f}\left(T_{f}+1\right)+t_{W}^{2} \frac{Y_{f}^{2}}{4}-s_{W}^{2} Q_{f}^{2}+\left(f \leftrightarrow f^{\prime}\right)\right]\left[T_{f^{\prime}}^{3} T_{f}^{3}+t_{W}^{2} \frac{Y_{f^{\prime}} Y_{f}}{4}\right]\right. \\
& +\left[-4 \ln \left(\frac{u}{s}\right)+2 \ln \left(\frac{u}{t}\right)+\ln \left(\frac{u}{t}\right) t_{W}^{2} Y_{f^{\prime}} Y_{f}\right] T_{f^{\prime}}^{3} T_{f}^{3}+\frac{3}{4} \ln \left(\frac{u}{t}\right) \delta_{I L} \delta_{J L} \\
& \left.+\ln \left(\frac{u}{t}\right)\left[t_{W}^{2} Y_{f^{\prime}} Y_{f}-4 s_{W}^{2} Q_{f^{\prime}} Q_{f}\right]\left[T_{f^{\prime}}^{3} T_{f}^{3}+t_{W}^{2} \frac{Y_{f^{\prime} Y_{f}}}{4}\right]\right\} L(s) l(s) A_{I J}^{f^{\prime} f}
\end{aligned}
$$

With the expression for the chiral amplitudes at hand, we can compute the leading and subleading logarithmic corrections to the basic observables for $e^{+} e^{-} \rightarrow f \bar{f}$ using standard formulae. Though the above approximation is not formally valid for small angles $\theta<M / \sqrt{s}$ we can integrate the differential cross section over all angles to get a result with the logarithmic accuracy. Let us, for example, consider the total cross sections of the quark-antiquark $/ \mu^{+} \mu^{-}$ production in the $e^{+} e^{-}$annihilation. In the two loop approximation, the leading and nextto-leading Sudakov corrections to the cross sections read

$$
\begin{aligned}
& \sigma / \sigma_{B}\left(e^{+} e^{-} \rightarrow Q \bar{Q}\right)=1+5.30 l(s)-1.66 L(s)-12.84 l(s) L(s)+1.92 L^{2}(s) \\
& \sigma / \sigma_{B}\left(e^{+} e^{-} \rightarrow q \bar{q}\right) \\
& \sigma / \sigma_{B}\left(e^{+} e^{-} \rightarrow \mu^{+} \mu^{-}\right)=1+20.54 l(s)-2.17 L(s)-53.72 l(s) L(s)+2.79 L^{2}(s)
\end{aligned}
$$

where $Q=u, c, t, q=d, s, b$, and we use $s_{W}^{2}=0.232$ for numerical estimates. Numerically, $L(s)=0.07(0.11)$ and $l(s)=0.014(0.017)$ respectively for $\sqrt{s}=1 \mathrm{TeV}$ and $2 \mathrm{TeV}$. Here $M=M_{W}$ has been chosen for the infrared cutoff and $g^{2} / 16 \pi^{2}=2.7 \cdot 10^{-3}$ for the $S U(2)$ coupling. For physical applications, the running of $g$ and $s_{W}$, the $W$ and $Z$ boson mass difference and the top quark mass effects are important in the one loop approximation. Fortunately the first order corrections are known exactly beyond the logarithmic approximation 25.

Clearly, for energies at 1 and $2 \mathrm{TeV}$ the two loop corrections are huge and amount up respectively to $5 \%$ and $7 \%$. There is a cancellation between the leading and subleading logarithms and for the above energy interval the subleading contribution even exceeds the leading one. The higher order leading and next-to-leading corrections however do not exceed $1 \%$ level. They can be in principle resummed using the formulae given above.

For completeness we give a numerical estimate of corrections to the cross section asymmetries. In the case of the forward-backward asymmetry (the difference of the cross section averaged over forward and backward semispheres with respect to the electron beam direction divided by the total cross section) we get

$$
A_{F B} / A_{F B}^{B}\left(e^{+} e^{-} \rightarrow Q \bar{Q}\right) \quad=1-1.23 l(s)-0.09 L(s)+0.11 l(s) L(s)+0.12 L^{2}(s)
$$




$$
\begin{aligned}
& A_{F B} / A_{F B}^{B}\left(e^{+} e^{-} \rightarrow q \bar{q}\right)=1+7.16 l(s)-0.14 L(s)-1.54 l(s) L(s)+0.02 L^{2}(s), \\
& A_{F B} / A_{F B}^{B}\left(e^{+} e^{-} \rightarrow \mu^{+} \mu^{-}\right)=1+5.48 l(s)-0.04 L(s)-6.39 l(s) L(s)+0.27 L^{2}(s) .
\end{aligned}
$$

For the left-right asymmetry (the difference of the cross sections of the left and right particles production divided by the total cross section) we obtain

$$
\begin{aligned}
& A_{L R} / A_{L R}^{B}\left(e^{+} e^{-} \rightarrow Q \bar{Q}\right)=1+10.17 l(s)-2.77 L(s)+6.38 l(s) L(s)-0.91 L^{2}(s) \\
& A_{L R} / A_{L R}^{B}\left(e^{+} e^{-} \rightarrow q \bar{q}\right) \\
& A_{L R} / A_{L R}^{B}\left(e^{+} e^{-} \rightarrow \mu^{+} \mu^{-}\right)=1+11.66 l(s)-1.08 L(s)+15.27 l(s) L(s)-0.77 L^{2}(s),
\end{aligned}
$$

Let us compare our results with results of previous analyses. Our result for the one loop double logarithmic contribution is in agreement with [16]. However the result for the one loop single infrared logarithmic contribution differs from [17]. The reason is that, in [17], only the diagrams with heavy virtual bosons have been taken into account. There is an infrared safe contribution of the diagram with the virtual massless photon where the heavy boson mass serves as an infrared regulator that should be taken into account to get a complete (exponential) result. In one-loop approximation, this contribution comes from the box diagrams with the photon and $Z$ boson running inside the loop. One of the two collinear regions of these diagrams (see Section 3 ) gives an infrared safe contribution that should be taken into account. The contribution from this diagram combined with real radiation has been obtained in analytical form in [26]. Note that neglecting contributions of this type leads also to the breakdown of the exponentiation of the double logarithms starting from the two-loop approximation 18.

Our result for the two-loop double logarithmic contribution is in agreement with [19]. On the other hand, the coefficients in front of the two-loop leading logarithms in eq. (53) with a few percent accuracy coincide with the result of [18] where the photon contributions were not considered. This is related to the fact that the virtual photon contribution not included to the result of [18] is suppressed by a small factor $s_{W}^{2}$.

Note that our analysis implies the resolution energy for the real photon emission to be smaller than the heavy boson mass. The above result for the cross section should be multiplied by the standard factor which takes into account the soft photon emission and the pure QED virtual corrections. This factor depends logarithmically on $s, \omega_{\text {res }}$ and the initial/final fermion masses but not on $M_{Z, W}$. If the resolution energy exceeds $M_{Z, W}$ the analysis is more complicated due to the fact that the radiation of real photons is not of Poisson type because of its non-Abelian $S U(2)_{L}$ component. A complete analysis of this problem in the double logarithmic approximation is given in ref. [19. However, as we have pointed out the effects of the non-Abelian component of the photon are numerically rather small.

\section{Conclusion}

In the present paper, we have analyzed the asymptotic behavior of the Abelian form factor and four fermion amplitude in the $S U(N)$ gauge theory in two standard variants of the 
Sudakov limit: with on-shell massless fermions and massive gauge bosons and with offshell massless fermions and massless gauge bosons. The generalized strategy of regions and dimensional regularization were used to obtain the asymptotic expansions of one-loop diagrams that determine the structure of the evolution equations for the amplitudes in the Sudakov limit up to the next-to-leading logarithmic approximation. By integrating these equations the next-to-leading logarithms were summed up. The method can be directly extended to the next-to-next-to-leading logarithms. To do this one can apply expansions of two-loop Feynman integrals within the strategy of regions (in the case of the form factor see examples of expansions of master scalar integrals in [13, 27]) and insert two-loop information into the evolution equations.

We have applied our results to the analysis of the electroweak corrections to the process of fermion-antifermion pair production in the $e^{+} e^{-}$annihilation. The two-loop leading and next-to-leading logarithmic corrections to the chiral amplitudes which are supposed to saturate the total two-loop electroweak corrections in $\mathrm{TeV}$ region have been obtained. The corresponding corrections to the total cross sections and asymmetries of the quark-antiquark and $\mu^{+} \mu^{-}$production in the $e^{+} e^{-}$annihilation have been found to be of a few percent magnitude at the energy of 1-2 TeV. The next-to-leading infrared logarithms are comparable and even exceed the leading ones at this scale.

\section{Acknowledgments}

The work by V.S. was supported by the Volkswagen Foundation, contract No. I/73611. The work by J.K. and A.P. was supported by the Volkswagen Foundation, by BMBF under grant BMBF-057KA92P and by DFG-Forschergruppe "Quantenfeldtheorie, Computeralgebra und Monte-Carlo-Simulationen" (DFG Contract KU502/6-1). The work by A.P. was supported by the Bundesministerium für Bildung und Forschung under Contract No. 05 HT9GUA 3, and by the European Commission through the Research Training Network Quantum Chromodynamics and the Deep Structure of Elementary Particles under Contract No. ERBFMRXCT980194. We are greatful to M. Beneke for careful reading of the manuscript. V.S. is thankful to the institute of Theoretical Particle Physics of the University of Karlsruhe for kind hospitality when this work was completed.

\section{References}

[1] V.V. Sudakov, Zh. Eksp. Teor. Fiz. 30 (1956) 87.

[2] R. Jackiw, Ann. Phys. 48 (1968) 292; 51 (1969) 575.

[3] J.M. Cornwall and G. Tiktopoulos, Phys. Rev. Lett. 35 (1975) 338; Phys. Rev. D13 (1976) 3370.

[4] J. Frenkel and J.C. Taylor, Nucl. Phys. B116 (1976) 185.

[5] V. Smilga Nucl.Phys. B161 (1979) 449. 
[6] J.C. Collins, Phys.Rev. D22 (1980) 1478; in Perturbative QCD, ed. A.H. Mueller, 1989, p. 573.

[7] A. Sen, Phys. Rev. D24 (1981) 3281.

[8] A. Sen, Phys. Rev. D28 (1983) 860.

[9] G. Korchemsky, Phys. Lett. B217 (1989) 330.

[10] G. Korchemsky, Phys. Lett. B220 (1989) 629.

[11] L. Magnea and G. Sterman, Phys. Rev. D42 (1990) 4222.

[12] M. Beneke and V.A. Smirnov, Nucl. Phys. B522 (1998) 321.

[13] V.A. Smirnov and E.R. Rakhmetov, Teor. Mat. Fiz. 120 (1999) 64; V.A. Smirnov, Phys. Lett. B465 (1999) 226.

[14] G. 't Hooft and M. Veltman, Nucl. Phys. B44 (1972) 189; C.G. Bollini and J.J. Giambiagi, Nuovo Cim. 12B (1972) 20.

[15] M. Kuroda, G. Moultaka and D. Schildknecht, Nucl. Phys. B350 (1991) 25;

G. Degrassi and A. Sirlin, Phys. Rev. D46 (1992) 3104;

M. Beccaria et al., Phys. Rev. D58 (1998) 093014.

[16] P. Ciafaloni and D. Comelli, Phys. Lett. B446 (1999) 278.

[17] M. Beccaria et al., Preprint PM/99-26, hep-ph/9906319.

[18] J.H. Kühn and A.A. Penin, Preprint TTP/99-28, hep-ph/9906545.

[19] V.S. Fadin, L.N. Lipatov, A.D. Martin and M. Melles, Preprint PSI PR-99-24, hep$\mathrm{ph} / 9910338$.

[20] G. Sterman Phys. Rev. D17 (1978) 2773;

S.Libby and G. Sterman Phys. Rev. D18 (1978) 3252;

A.H. Mueller, Phys. Rep. 73 (1981) 35.

[21] J. Frenkel and R. Meuldermans, Phys. Lett. B65 (1976) 64.

[22] J. Frenkel, Phys. Lett. B65 (1976) 383.

[23] D. Amati, R. Petronzio and G. Veneziano, Nucl. Phys. B146 (1978) 29.

[24] J. Botts and G. Sterman, Nucl. Phys. B325 (1989) 62;

N. Kidonakis, G Odereda and G. Sterman, Nucl. Phys. B531 (1998) 365.

[25] W. Beenakker, W. Hollik and Van der Mark, Nucl. Phys. B365 (1991) 24. 
[26] J.H. Kühn and R.G. Stuart, Phys. Lett. B200 (1987) 360.

[27] V.A. Smirnov, Phys. Lett. B404 (1997) 101. 